\title{
Evaluation of the Relationship between Mucosal Diseases and Faecal Calprotectin Levels in Children who Underwent Upper Gastrointestinal System Endoscopy
}

\author{
(1) Maşallah Baran1, (1) Yeliz Çağan Appak², (1) Fatma Demet Arslan33, (1) Dudu Solakoğlu Kahraman4, \\ ๑ Miray Karakoyun2, ๑ Inanç Karakoyun33, ๑ Büşra Emir5, ๑ Gülden Diniz 4 \\ 1̇zmir Katip Çelebi University Faculty of Medicine, Department of Pediatric Gastroenterology Hepatology and Nutrition, İzmir, Turkey \\ 2University of Health Science, Tepecik Training and Research Hospital, Clinic of Pediatric Gastroenterology Hepatology and Nutrition, İzmir, Turkey \\ 3 University of Health Science, Tepecik Training and Research Hospital, Clinic of Biochemistry, İzmir, Turkey \\ ${ }^{4}$ University of Health Science, Tepecik Training and Research Hospital, Clinic of Pathology, Izmir, Turkey \\ 5 İzmir Katip Çelebi University Faculty of Medicine, Department of Biostatistics, İzmir, Turkey
}

\section{ABSTRACT}

Aim: In this study, the correlation of faecal calprotectin (FC) levels with endoscopic and histopathological findings was evaluated in children who underwent upper gastrointestinal system (UGIS) endoscopy.

Materials and Methods: The patients aged 4-18 years admitted to our endoscopy unit were included in this study. Faecal samples were collected on the day participants presented for the study. A calprotectin assay was performed on the faecal samples using enzyme-linked immunosorbent assay kits. FC levels were compared with those of children with normal histopathology.

Results: The FC levels of 112 children included in this study were evaluated according to their histopathological diagnoses. Their FC levels were not found to be significantly higher than those in healthy children. No significant difference was found in the FC levels of participants with or without signs of inflammation in the esophagus, stomach and duodenum. In those participants with chronic gastritis, Helicobacter pylori (H. pylori) gastritis, reflux esophagitis or celiac disease, FC levels above cut-off values were observed. However, no significant difference was found in those participants with normal endoscopic findings. There was no significant difference in FC levels between similar regions of the UGIS with or without signs of inflammation. A positive correlation was discovered between eosinophil counts and FC levels in patients with $H$. pylori gastritis and esophagitis.

Conclusion: The diagnostic value of FC levels in UGIS diseases was not sufficient to establish a definitive diagnosis in our study.

Keywords: Calprotectin, children, gastritis, esophagitis

\section{Introduction}

Faecal calprotectin (FC) found in neutrophils is a complex protein bound to calcium and zinc, and it is resistant to enzymatic degradation and unaffected by medications or diet. It is released into the lumen as a result of alterations in the intestinal barrier induced by inflammation $(1,2)$. In childhood, higher levels of FC, mainly in inflammatory bowel disease (IBD) and Henoch Schönlein vasculitis have 
been reported $(1,3)$. In some studies, although slightly higher levels of FC have been reported at the time of celiac disease diagnosis, these increased FC levels have no diagnostic value (4-6).

FC has been evaluated in other upper gastrointestinal system (UGIS) diseases such as reflux esophagitis, Helicobacter pylori (H. pylori) gastritis, eosinophilic enteropathy and eosinophilic esophagitis in children, but there are an inadequate number of studies on these. It has been indicated that FC plays a role in the differentiation between organic and functional bowel diseases of the gastrointestinal system and is associated with findings of endoscopic and histopathological inflammation $(1,7)$. In this study, endoscopic and histopathological findings and the relationship between eosinophil and neutrophil counts in biopsy materials were evaluated in pediatric patients undergoing UGIS endoscopy.

\section{Materials and Methods}

Patients aged 4-18 years admitted to Tepecik Training and Research Hospital Pediatric Gastroenterologic Endoscopy unit between June 2017 and January 2018 were included in this study. Informed consent was obtained from the family and patients. This study was approved by the local Ethics Committee (approval number: 77- 21.4.2016).

Our study population consisted of patients scheduled for UGIS endoscopy with presumptive diagnoses of gastritisesophagitis based on complaints of chronic dyspepsia, vomiting, nausea or epigastric pain and a presumptive diagnosis of celiac disease or tissue transglutaminase (TTG) positivity (>20 RU/mL). Participants who complained of longterm diarrhea with TTC IgA negativity, TTC IgC negativity, or those with normal colonoscopy and histopathology findings were also included in the study. Cases of foreign body swallowing, varicose vein control, gastrointestinal bleeding, patients requiring endoscopic dilatation, recent users of nonsteroidal anti-inflammatory drugs, those with a detected infection or IBD were not included in this study (2).

Parents were asked to provide their child's faecal sample on the morning of endoscopy after informed consent for the study was obtained. Faecal sampling and the endoscopic procedure were performed on the same day. The same pediatric gastroenterology team performed all endoscopic procedures. Biopsies were taken with endoscopic forceps from the duodenum, duodenal bulb, antrum of the stomach, and esophagus in the same session.

The native stool samples (50-100 mg) were homogenized according to the manufacturer's directions using the Smart-
Prep faecal sample preparation kit (Bühlmann Laboratories AG, Schönenbuch, Switzerland). The homogenized stool samples were transferred into plastic tubes and stored at $-20{ }^{\circ} \mathrm{C}$ until analyzed. Calprotectin concentrations in fecal samples were measured using enzyme-linked fCAL ${ }^{\circledR}$ immunosorbent assay kits (Bühlmann Diagnostics Corp., Amherst, NH, USA). The lowest and the highest values within the working range were considered to be 30 and $1.800 \mu \mathrm{g} / \mathrm{G}$, respectively. The results were represented as $\mu \mathrm{g} / \mathrm{G}$. The cut-off value recommended by the manufacturer was $50 \mu \mathrm{g} / \mathrm{G}$ for children aged 4-17 years, regardless of gender (8).

All biopsy specimens were evaluated by the same pathology team. Gastric endoscopic biopsy samples were evaluated histopathologically with the updated Sydney classification and grading system, and the gastritis and $H$. pylori status of each patient was determined (9). Using this classification, chronic inflammation, neutrophil activity, glandular atrophy, intestinal metaplasia and $H$. pylori density were graded and evaluated. Intestinal metaplasia was histochemically assessed by pediatric appendicitis scoreAlcian Blue staining in addition to histomorphologic studies. The presence of $\mathrm{H}$. pylori was determined by Toluidine blue staining. Gastritis was classified as mild, moderate or severe according to $H$. pylori density $(10,11)$.

In addition, eosinophils, leukocytes and neutrophils were counted in the lamina propria of gastric biopsies under 400x magnification. Diagnosis of eosinophilic gastritis was made based on the presence of more than 30 eosinophils in gastric biopsy materials under 400x magnification $(12,13)$. Bulbus and duodenum villus to crypt ratio, crypt hyperplasia, intraepithelial lymphocyte count, neutrophil and eosinophil leukocyte counts in the lamina propria and epithelium were evaluated with hematoxylin and eosin (H\&E) and CD3 stained sections of biopsy specimens $(14,15)$.

The FC levels in participants who were diagnosed based on histopathological and endoscopic findings of the esophagus, stomach, bulbus and duodenum were compared with the FC levels of participants with normal histopathology. The relationship between FC values and eosinophil and neutrophil counts in the biopsy samples was also evaluated.

\section{Statistical Analysis}

All statistical analyses were performed using the IBM SPSS Statistics 25 package program (IBM Corp., Armonk, New York, USA). Data are presented as count (n), percent $(\%)$, mean and standard deviation ( $\bar{x} \pm s d)$, minimummaximum value and median (25\%-75\% quartiles). Shapiro- 
Wilk's test was used and a histogram and Q-Q plot were examined to assess the data normality. Mann-Whitney $U$ test was used to compare the differences between two groups for continuous variables. Kruskal-Wallis analysis was used for comparisons of more than two groups. The relationship between variables was evaluated by Spearman correlation analysis. Receiver operating characteristic curves were used to determine the discriminative power of the FC levels in the diagnosis of histopathological findings (normal, abnormal). A $p$ value $<0.05$ was accepted as statistically significant.

\section{Results}

Of the 112 patients included in the study, 68\% were female $(n=77)$ with a mean age of $12.8 \pm 4.5$ years. Endoscopy was planned based on the following indications: gastric complaints (59\%; $n=67)$, persistent reflux symptoms (12.5\%; $n=14)$, TTC IgA-IgG positivity $(18 \% ; n=20)$ and other causes that included chronic epigastric pain, chronic diarrhea or dysphagia $(10 \% ; n=11)$. As a result of UGIS endoscopy, diagnoses were as follows: chronic gastritis/ $H$. pylori gastritis $(67.8 \% ; n=76)$, biliary reflux $(2.7 \% ; n=3)$, celiac enteropathy (13.4\%; $n=15)$, duodenitis-bulbitis $(4.4 \%$; $\mathrm{n}=5)$, reflux esophagitis $(6.2 \% ; n=7)$ and eosinophilic esophagitis $(0.9 \% ; n=1)$, while $4.5 \%(n=5)$ had normal endoscopic findings.

The FC levels in 95 cases (85\%) with abnormal histopathologic findings and 17 cases (15\%) with normal histopathological findings are shown in Table I. In one of the two cases with eosinophilic esophagitis, the FC level was found to be $850 \mu \mathrm{g} / \mathrm{G}$, while the other case had FC levels within normal limits. In one participant with severe eosinophilic duodenitis and ulceration, the FC level was found to be $1.200 \mu \mathrm{g} / \mathrm{G}$. As a result, when FC levels were evaluated according to histopathological diagnosis, there was no significant difference in the FC levels between the biopsy specimens of cases with or without abnormal findings (Figure 1, Table I).

No significant difference in FC levels between similar regions of the UGIS with or without signs of inflammation was detected (Table II). A positive correlation was found

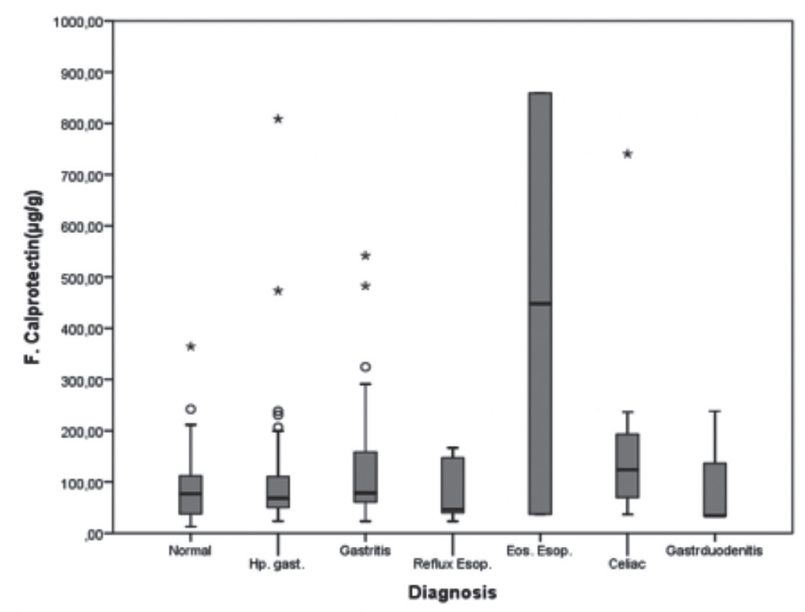

Figure 1. Fecal calprotectin levels according to histopathological diagnoses

Hp. gast.: Helicobacter pylori gastritis, Esop.: Esophagitis, Eos.: Eosinophilic

Table I. Comparison of fecal calprotectin levels according to histopathological diagnosis

\begin{tabular}{|c|c|c|c|c|c|c|c|}
\hline & Diagnosis & $\mathbf{n}$ & $M\left(Q_{1}-Q_{3}\right)$ & Min-max & Mean Rank & Test Statistics & $\mathbf{p}^{+}$ \\
\hline \multirow[t]{9}{*}{ FC } & Normal & 17 & $\begin{array}{l}108 \pm 94.7 \\
76.8(36.2-156.5)\end{array}$ & $12.7-364.4$ & 53.09 & \multirow{9}{*}{8.933} & \multirow{9}{*}{0.348} \\
\hline & Hp. Gast. & 36 & $\begin{array}{l}116.2 \pm 146.5 \\
68.4(49.5-112.9)\end{array}$ & $23.5-808.5$ & 51.38 & & \\
\hline & Gastritis & 33 & $\begin{array}{l}131.3 \pm 124.4 \\
78.5(56.7-163.6)\end{array}$ & $23.2-541.8$ & 60.12 & & \\
\hline & Reflux Esop. & 5 & $\begin{array}{l}84.6 \pm 66.7 \\
46.4(31.6-156.8)\end{array}$ & $23.2-166.5$ & 43.10 & & \\
\hline & Eos. Esop. & 2 & $\begin{array}{l}448.1 \pm 581.1 \\
448.1(37.20--)\end{array}$ & $37.2-859$ & 64.50 & & \\
\hline & Bulbitis & 1 & - & $58.1-58.1$ & 34.00 & & \\
\hline & Duodenit-Eos. & 1 & - & $1264-1264$ & 112.00 & & \\
\hline & Celiac & 14 & $\begin{array}{l}168.3 \pm 176 \\
124(68.3-196.6)\end{array}$ & $36.6-740.5$ & 69.89 & & \\
\hline & Gastroduodenitis & 3 & $\begin{array}{l}102.1 \pm 118 \\
34.2(33.8--)\end{array}$ & $33.8-238.4$ & 41.00 & & \\
\hline
\end{tabular}


between eosinophil and neutrophil counts in the esophagus, antrum, bulbus and duodenum ( $r=0.483,0.687,0.392$, and 0.368 , respectively) $(p<0.001)$. When the correlation between eosinophil and neutrophil counts with FC levels in the biopsy specimens was examined, positive correlation was found between eosinophil counts and FC in patients with H. pylori gastritis and reflux esophagitis (Table III).

Finally, normal and abnormal endoscopic and histopathological findings were compared based on the FC cut-off level of $50 \mu \mathrm{g} / \mathrm{G}$. The sensitivity and specificity of this cut-off value was determined to be $75 \%$ and $35 \%$, respectively (Figure 2 ).

\section{Discussion}

FC is a marker that assists in the differentiation of diseasespecific and functional gastrointestinal system diseases, showing mucosal inflammation of the gastrointestinal system lumen. Although FC is resistant to bacterial enzymes and intestinal proteases, it appears that FC does not have a practical role in UGIS diseases in children (16-18).

In this study, endoscopic and histopathological findings and FC measurements of children with UGIS were evaluated in combination. In participants with UGIS pathologies, although the mean FC levels were above the cut-off value, no significant increase was observed compared to participants with normal histopathological findings. Different results have been reported in the literature regarding FC levels in different UGIS diseases (19). It has been reported that FC levels in peptic ulcer disease and erosive gastritis in adults are higher compared to normal endoscopic findings, with $60 \%$ sensitivity and $81 \%$ specificity in the prediction of endoscopic findings (20). Since we did not find gastric peptic ulcer disease and erosive gastritis in our study participants, data related to these diseases could not be presented. In another study performed in children, a significant correlation was found between FC level and gastritis (severity and

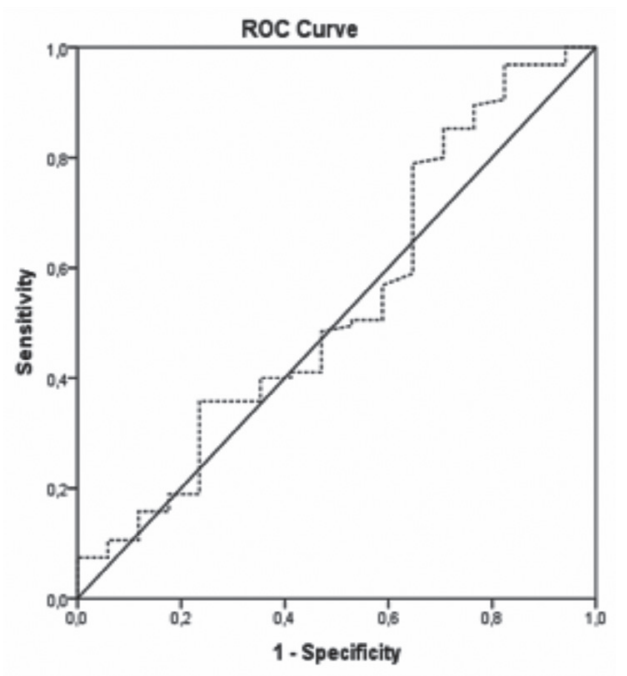

Figure 2. Evaluation of the level of fecal calprotectin and pathologic findings in histopathology and endoscopy of the upper gastrointestinal tract

Area under the ROC curve (AUC): 0.536 (95\% Confidence Interval; 0.439 to 0.631 ); Significance level $p$ (Area $=0.50$ ): 0.664 ROC: Receiver operating characteristic

\begin{tabular}{|c|c|c|c|c|c|c|c|}
\hline $\begin{array}{l}\text { Parts of Upper } \\
\text { Intestine }\end{array}$ & Histopathology & $\mathbf{n}$ & $M\left(Q_{1}-Q_{3}\right)$ & Min-max & $\begin{array}{l}\text { Mean } \\
\text { Rank }\end{array}$ & $\begin{array}{l}\text { Test } \\
\text { Statistics }\end{array}$ & $\mathrm{p}^{\dagger}$ \\
\hline \multirow[t]{2}{*}{ Esophagus } & Normal & 91 & $\begin{array}{l}121.2 \pm 136.1 \\
72.8(50.3-132.8)\end{array}$ & $12.7-808$ & 52.7 & \multirow{2}{*}{-1.018} & \multirow{2}{*}{0.309} \\
\hline & Abnormal & 16 & $\begin{array}{l}251.4 \pm 345.8 \\
112(41.6-245.4) \\
\end{array}$ & $23.2-1264$ & 61.3 & & \\
\hline \multirow[t]{2}{*}{ Antrum } & Normal & 25 & $\begin{array}{l}145.3 \pm 192.5 \\
76(37.2-183.9)\end{array}$ & $12.7-859$ & 51.6 & \multirow{2}{*}{-0.685} & \multirow{2}{*}{0.493} \\
\hline & Abnormal & 85 & $\begin{array}{l}139.4 \pm 182.8 \\
76.8(54.3-158.3)\end{array}$ & $23.2-1264$ & 56.6 & & \\
\hline \multirow[t]{2}{*}{ Duodenum } & Normal & 87 & $\begin{array}{l}129.77 \pm 150.6776 .8(47.4- \\
158.3)\end{array}$ & $12.7-859$ & 55.2 & \multirow{2}{*}{-0.807} & \multirow{2}{*}{0.420} \\
\hline & Abnormal & 25 & $\begin{array}{l}174.7 \pm 267.3 \\
72.8(61.1-169.7)\end{array}$ & $33.8-1264$ & 61.1 & & \\
\hline \multirow[t]{2}{*}{ Duodenal- Bulb } & Normal & 86 & $\begin{array}{l}132.6 \pm 150.5 \\
76.8(50-160.3)\end{array}$ & $12.7-859$ & 56.8 & \multirow{2}{*}{-0.522} & \multirow{2}{*}{0.601} \\
\hline & Abnormal & 25 & $\begin{array}{l}140.2 \pm 242.8 \\
69.9(37.2-130.7)\end{array}$ & $23.2-1264$ & 53.1 & & \\
\hline
\end{tabular}


Baran et al.

Faecal Calprotectin Levels in Children

\begin{tabular}{|c|c|c|c|c|c|c|c|c|}
\hline & Upper GI parts & & Normal -FC & $\begin{array}{l}\text { Hp. Gastritis } \\
\text { FC }\end{array}$ & $\begin{array}{l}\text { Cr. Gastritis } \\
\text { FC }\end{array}$ & $\begin{array}{l}\text { Reflux Esop.- } \\
\text { FC }\end{array}$ & Celiac- FC & $\begin{array}{l}\text { Gastro- } \\
\text { duodenitis -FC }\end{array}$ \\
\hline \multirow{12}{*}{ 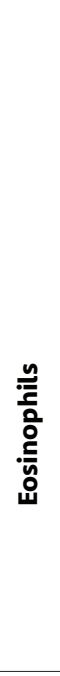 } & \multirow[t]{3}{*}{ Esophagus } & $r_{s}$ & 0.077 & - & 0.031 & 0.894 & - & - \\
\hline & & $\mathrm{p}$ & 0.802 & - & 0.870 & 0.041 & - & - \\
\hline & & $\mathrm{n}$ & 13 & 35 & 31 & 5 & 12 & 3 \\
\hline & \multirow[t]{3}{*}{ Antrum } & $r_{s}$ & 0.000 & 0.353 & -0.157 & 0.289 & 0.559 & 0.500 \\
\hline & & $\mathrm{p}$ & 1.000 & 0.044 & 0.417 & 0.638 & 0.118 & 0.667 \\
\hline & & $\mathrm{n}$ & 14 & 33 & 29 & 5 & 9 & 3 \\
\hline & \multirow[t]{3}{*}{ Duodenal-Bulb } & $r_{s}$ & -0.306 & 0.033 & -0.140 & 0.200 & 0.258 & -0.500 \\
\hline & & $\mathrm{p}$ & 0.310 & 0.854 & 0.486 & 0.800 & 0.471 & 0.667 \\
\hline & & $\mathrm{n}$ & 13 & 33 & 27 & 4 & 10 & 3 \\
\hline & \multirow[t]{3}{*}{ Duodenum } & $r_{s}$ & -0.268 & 0.123 & -0.164 & -0.738 & 0.498 & -1.000 \\
\hline & & $\mathrm{p}$ & 0.355 & 0.497 & 0.415 & 0.262 & 0.173 & \\
\hline & & $\mathrm{n}$ & 14 & 33 & 27 & 4 & 9 & 3 \\
\hline \multirow{12}{*}{ 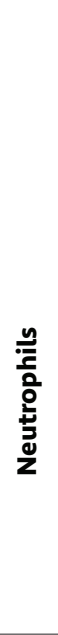 } & \multirow[t]{3}{*}{ Esophagus } & $r_{s}$ & 0.077 & 0.209 & -0.024 & - & - & 0.866 \\
\hline & & $\mathrm{p}$ & 0.802 & 0.244 & 0.897 & - & - & 0.333 \\
\hline & & $\mathrm{n}$ & 13 & 33 & 31 & 5 & 11 & 3 \\
\hline & \multirow[t]{3}{*}{ Antrum } & $r_{s}$ & -0.047 & 0.072 & -0.143 & 0.289 & 0.453 & 0.866 \\
\hline & & $p$ & 0.881 & 0.697 & 0.478 & 0.638 & 0.221 & 0.333 \\
\hline & & $\mathrm{n}$ & 14 & 32 & 27 & 5 & 9 & 3 \\
\hline & \multirow[t]{3}{*}{ Duodenal-Bulb } & $r_{s}$ & 0.437 & -0.202 & 0.010 & -0.738 & 0.364 & 0.000 \\
\hline & & $\mathrm{p}$ & 0.136 & 0.284 & 0.960 & 0.262 & 0.301 & 1.000 \\
\hline & & $n$ & 13 & 30 & 27 & 4 & 10 & 3 \\
\hline & \multirow[t]{3}{*}{ Duodenum } & $r_{s}$ & 0.044 & -0.105 & -0.001 & -0.105 & 0.143 & -0.500 \\
\hline & & $\mathrm{p}$ & 0.881 & 0.581 & 0.995 & 0.895 & 0.694 & 0.667 \\
\hline & & & 11 & 1 & 1 & 1 & 1 & 1 \\
\hline
\end{tabular}

inflammation grade) and colonization of $H$. pylori (21). In our study, there was no significant difference between FC levels in participants with or without $H$. pylori chronic gastritis. In participants with $\mathrm{H}$. pylori-associated gastritis, FC was found to be above the cut-off value. However, no statistically significant differences were observed between those patients with normal gastric mucosa and those with H. pylori-positive gastritis. In another study, the FC was not found to be significantly higher among participants with histologically active or non-active gastritis than among healthy controls (21).

Increased FC levels have been demonstrated in celiac disease and are also associated with villus atrophy and a gluten-free diet $(5,6)$. However, it was reported that these high values have no diagnostic specificity (19). In our study, no significant difference was observed in the FC levels of children with a diagnosis of celiac disease compared to patients with normal duodenal histopathology. This is an expected condition when we consider that the main histopathological element in this condition is intraepithelial lymphocyte infiltration.

Higher FC levels have been reported in people with allergic colitis and cow's milk protein allergy $(16,22)$. Our study population did not contain any cases of cow's milk protein allergy. However, higher FC levels were detected in one participant with severe eosinophilic esophagitis, and another with dense eosinophilic inflammation and an ulcer in the duodenum. When the biopsy materials of these two cases were examined, it was observed that the number of neutrophils increased in parallel with an increase in the 
number of eosinophils. Neutrophil counts were found to be within normal limits in another participant with eosinophilic esophagitis and a normal FC level. In our study, it was determined that eosinophil and neutrophil counts in tissue samples were moderately, and also positively, correlated. This may explain increased FC levels in cases with severe eosinophilic inflammation. Owing to the limited number of participants with eosinophilic esophagitis and eosinophilic enteropathy in our study, it is not possible to make a definitive interpretation about the relationship between these diseases and FC levels. Another study reported that FC levels increased in parallel with disease severity in children with IBD, but FC levels were within normal limits in eosinophilic and lymphocytic colitis (23).

In one study evaluating endoscopic findings in UGIS and FC levels in children, it was stated that there was no correlation between FC values and esophageal pathologies, and there was a lack of any marker for esophageal pathologies (21). In our study, we found no significant elevation of FC levels in reflux esophagitis.

Intensive ulceration and inflammation in UGIS disease in children is a rare condition. It has also been reported that the level of neutrophils in the lumen is associated with FC levels $(2,23)$. In two patients with critically high FC values, severe mucosal inflammation and ulceration was observed. This suggests that inflammation in the tissue may be associated with an increase in the release of neutrophils into the lumen. A significant elevation of FC levels in adults with peptic ulcer disease and erosive gastritis compared to patients with normal endoscopic findings suggests this condition (20). In the cohort study performed among pediatric patients, the sensitivity and specificity of IBD were found to be $98 \%$ and $84 \%$, respectively. In this study, the sensitivity and specificity of FC values above the cut-off value were determined to be $75 \%$ and $35 \%$, respectively.

\section{Conclusion}

As a result, it is important to formulate diagnostic markers for UGIS diseases without resorting to invasive procedures such as UGIS endoscopy, which requires sedation, especially for pediatric patients. However, in our study, although FC levels were found to be slightly elevated in UGIS diseases, their diagnostic value was not sufficient to establish a diagnosis. FC levels should be studied in a larger series of patients with eosinophilic esophagitis and enteropathy.

\section{Ethics}

Ethics Committee Approval: This study was approved by the local ethics committee (approval number: 7721.4.2016).
Informed Consent: Informed consent was obtained from the family and patients.

Peer-review: Enternally and internally peer-reviewed.

\section{Authorship Contributions}

Surgical and Medical Practices: M.B., Y.Ç.A., G.D., Concept: M.B.,Y.Ç.A., Design: M.B., Y.Ç.A., M.K., Data Collection or Processing: M.B., Y.Ç.A., M.K., F.D.A., I.K., D.S.K., B.E., Analysis or Interpretation: M.B., Y.Ç.A., F.D.A., I.K., D.S.K., B.E., G.D., Literature Search: M.B., Y.Ç.A, B.E., Writing: M.B., Y.Ç.A, B.E., G.D.

Conflict of Interest: No conflict of interest was declared by the authors.

Financial Disclosure: The authors declared that this study was funded by İzmir Katip Çelebi University Scientific Research Foundation.

\section{References}

1. Holtman GA, Lisman-van Leeuwen Y, Kollen BJ, et al. Diagnostic Accuracy of Fecal Calprotectin for Pediatric Inflammatory Bowel Disease in Primary Care: A Prospective Cohort Study. Ann Fam Med 2016; 14:437-45.

2. Waugh N, Cummins E, Royle $P$, et al. Faecal calprotectin testing for differentiating amongst inflammatory and noninflammatory bowel diseases: systematic review and economic evaluation. Health Technol Assess 2013; 17:xv-xix, 1-211.

3. Kanik A, Baran M, Ince FD, et al. Faecal calprotectin levels in children with Henoch-Schönlein purpura: is this a new marker for gastrointestinal involvement? Eur I Gastroenterol Hepatol $2015 ; 27: 254-8$.

4. Biskou O, Gardner-Medwin I, Mackinder M, et al. Faecal Calprotectin in Treated and Untreated Children With Coeliac Disease and Juvenile Idiopathic Arthritis. I Pediatr Gastroenterol Nutr 2016; 63:e112-5.

5. Balamtekin N, Baysoy G, Uslu N, et al. Fecal calprotectin concentration is increased in children with celiac disease: relation with histopathological findings. Turk / Gastroenterol 2012; 23:503-8.

6. Ertekin V, Selimoğlu MA, Turgut A, Bakan N. Fecal calprotectin concentration in celiac disease. I Clin Gastroenterol 2010; 44:544-6.

7. Siddiqui I, Majid H, Abid S. Update on clinical and research application of fecal biomarkers for gastrointestinal diseases. World I Gastrointest Pharmacol Ther 2017; 8:39-46.

8. Fagerberg UL, Lööf L, Myrdal U, Hansson LO, Finkel Y. Colorectal inflammation is well predicted by fecal calprotectin in children with gastrointestinal symptoms. I Pediatr Gastroenterol Nutr 2005; 40:450-5.

9. Hassan TMM, Al-Najjar Sl, Al-Zahrani IH, Alanazi FIB, Alotibi MG. Helicobacter pylori chronic gastritis updated Sydney grading in relation to endoscopic findings and $\mathrm{H}$. pylori IgC antibody: diagnostic methods. I Microsc Ultrastruct 2016; 4:16774. 
10. Dixon MF, Genta RM, Yardley JH, Correa P. Classification and grading of gastritis. The updated Sydney System. Am I Surg Pathol 1996; 20:1161-81.

11. Dixon MF, Genta RM, Yardley JH, Correa P. Histological classification of gastritis and Helicobacter pylori infection: an agreement at last? The International Workshop on the Histopathology of Gastritis. Helicobacter 1997; 2:17-24.

12. Posten S, Adamiak T, Jensen M. Pediatric Eosinophilic Esophagitis. SD Med 2018; 71:362-6.

13. Dellon ES, Liacouras CA, Molina-Infante ), et al. Updated International Consensus Diagnostic Criteria for Eosinophilic Esophagitis: Proceedings of the AGREE Conference. Gastroenterology 2018; 155:1022-33.

14. Lemale J, Dainese L, Tounian P. Eosinophilic gastroenteritis and colitis in pediatric patients: Increasingly frequent diseases. Arch Pediatr 2015; 22:769-77.

15. Collins MH, Capocelli K, Yang GY. Eosinophilic Gastrointestinal Disorders Pathology. Front Med 2018; 4:261.

16. Canani RB, Terrin G, Rapacciuolo L, et al. Faecal calprotectin as reliable non-invasive marker to assess the severity of mucosal inflammation in children with inflammatory bowel disease. Dig Liver Dis 2008; 40:547-53.

17. Aadland E, Fagerhol MK. Fecal calprotectin: a marker of inflammation throughout the intestinal tract. Eur Gastroenterol Hepatol 2002; 14:823-5.
18. Fagerberg UL, Loof L, Lindholm J, Hansson LO, Finkel Y. Fecal calprotectin: a quantitative marker of colonic inflammation in children with inflammatory bowel disease. I Pediatr Gastroenterol Nutr 2007; 45:414-20.

19. Montalto M, Santoro L, Curigliano V, et al. Fecal calprotectin concentrations in untreated coeliac patients. Scand I Gastroenterol 2007; 42:957-61.

20. Manz M, Burri E, Rothen $C$, et al. Value of fecal calprotectin in the evaluation of patients with abdominal discomfort: an observational study. BMC Gastroenterol 2012; 12:5.

21. Pedram A, Vahidreza A, Bahram N, et al. Relationship Between Fecal Calprotectin and Upper Endoscopy Findings in Children With Upper Gastrointestinal Symptoms. Iran I Pediatr 2017; 27:e8658

22. Beşer OF, Sancak $S$, Erkan $T$, Kutlu $T$, Çokuğraş $H$, Çokuğraş FÇ. Can Fecal Calprotectin Level Be Used as a Markers of Inflammation in the Diagnosis and Follow-Up of Cow's Milk Protein Allergy? Allergy Asthma Immunol Res 2014; 6:33-8.

23. Komraus M, Wos H, WiecekS, KajorM, Grzybowska-Chlebowczyk $U$. Usefulness of Faecal Calprotectin Measurement in Children with Various Types of Inflammatory Bowel Disease. Mediators Inflamm 2012; 2012:608249. 